\title{
ICME Observations During the Ulysses Fast Latitude Scan
}

\author{
R. J. Forsyth*, A. Rees*, D. B. Reisenfeld ${ }^{\dagger}$, S. T. Lepri ${ }^{\mathrm{Il}}$, and T. H. Zurbuchen ${ }^{\mathbb{I I}}$ \\ *The Blackett Laboratory, Imperial College, London SW7 2BW, UK \\ ${ }^{+}$Los Alamos National Laboratory, Los Alamos, NM 87544, USA \\ ${ }^{\pi}$ University of Michigan, Ann Arbor, MI 48109, USA
}

\begin{abstract}
Between November 2000 and October 2001 the Ulysses spacecraft performed a fast traversal of the heliospheric latitudes between $80^{\circ} \mathrm{S}$ and $80^{\circ} \mathrm{N}$, a period close to the activity maximum of the current solar cycle. This paper provides an overview of the Ulysses observations of the transient solar wind structures associated with coronal mass ejections (ICMEs) during this period. Compared to the previous Ulysses fast latitude scan near solar minimum in 1995, many more ICME related signatures were observed in the present data set. Events were encountered spread over the full latitude range between $80^{\circ} \mathrm{S}$ and $80^{\circ} \mathrm{N}$. Those at high northern latitudes, where fast solar wind from a northern polar coronal hole had become re-established, were of the over-expanding type first identified in Ulysses data at midlatitudes near solar minimum. The signatures of these events and their latitude dependence are discussed and some ongoing and possible future studies with this data set are described.
\end{abstract}

\section{INTRODUCTION}

Coronal mass ejections (CMEs) are explosive eruptions of plasma from regions of the solar atmosphere that were previously magnetically closed, seen to dramatic effect by space-borne coronagraph instruments. These CMEs propagate out into the heliosphere embedded in and interacting with the solar wind. Here, their markedly different properties from the ambient solar wind lead to their identification as they pass over in-situ interplanetary spacecraft. The aim of this paper is to provide a first report and overview of the Ulysses observations of the transient solar wind structures associated with coronal mass ejections (known as ICMEs) during the recent fast latitude scan in 2000-2001.

The fast latitude scans are defined as the perihelion phases of the Ulysses orbit during which the spacecraft traverses heliographic latitudes between $80.2^{\circ} \mathrm{S}$ and $80.2^{\circ} \mathrm{N}$, with a perihelion of $1.3 \mathrm{AU}$, in a little over 10 months. Trajectory information for the recent fast scan, which took place between November 24 (day 329) 2000 and October 11 (day 284) 2001, is provided in Figure 1. This was the second such traversal of Ulysses through this latitude range. Whereas the first in 1995 took place during a period approaching solar activity minimum, the present fast latitude scan occurred close to the maximum of the present solar cycle (no. 23). Since CME occurrence rates have been shown to be a strong function of the solar cycle [1], as well as their tendency to occur at higher latitudes [2], we would expect this to be a prime period for their study. This data set thus provides unique opportunities for studying the latitude dependence of ICME occurrence and physical signatures. In this paper we first provide a catalogue of the well defined ICME events identified by the Ulysses instruments during the fast latitude scan and discuss latitude dependencies, including example events. Figure 1 also shows that for much of the fast latitude scan Ulysses was located in a direction off the west limb of the Sun as seen from the Earth. We explore the resulting opportunities for correlative studies linking Ulysses in-situ ICMEs with LASCO coronagraph [3] observations from SOHO.

\section{ICME OBSERVATIONS}

We have endeavored to identify and catalogue all the ICME events observed by Ulysses during the fast latitude scan. To do this we have employed data from three instruments, the magnetometer [4], the SWOOPS 
ion and electron instruments [5] and the solar wind composition instrument (SWICS) [6]. The list of events is presented in Table 1 along with a tabulation of the ICME signatures shown by each. It is well documented in the literature that ICMEs often have remarkably different magnetic field and plasma signatures from the solar wind in which they are embedded [e.g. 7]. Those referred to in Table 1 are, by column, counter-streaming suprathermal electrons (CSE), smooth magnetic field rotations consistent with flux rope-like field structures (FR), low plasma beta (Beta), whether the event is driving a shock wave (Sh) as it propagates through the solar wind, low proton thermal temperature (Tp), enhanced helium (alpha/ proton) abundance $(\mathrm{He})$, existence of high iron charge states $(\mathrm{Fe})$, and enhanced $\mathrm{O}^{7+} / \mathrm{O}^{6+}$ ratio $(\mathrm{O} 7 / \mathrm{O} 6)$ when compared to the surrounding solar wind. The events were mostly first selected by searching for at least two from among CSE signatures, magnetic field rotations, low beta, and enhanced He, although in one case the initial identification came from the charge state data. We then checked which of the other signatures were present. In general, ICME identification is made harder by the fact that not all ICMEs exhibit all the above signatures and also that the majority of these signatures can also arise from other causes. Thus the list presented here represents what we believe to be a complete set of the ICMEs identifiable from the Ulysses data under study, but cannot be absolutely guaranteed to be so.

For some of the signatures a few additional words of explanation are necessary. Of those events listed as

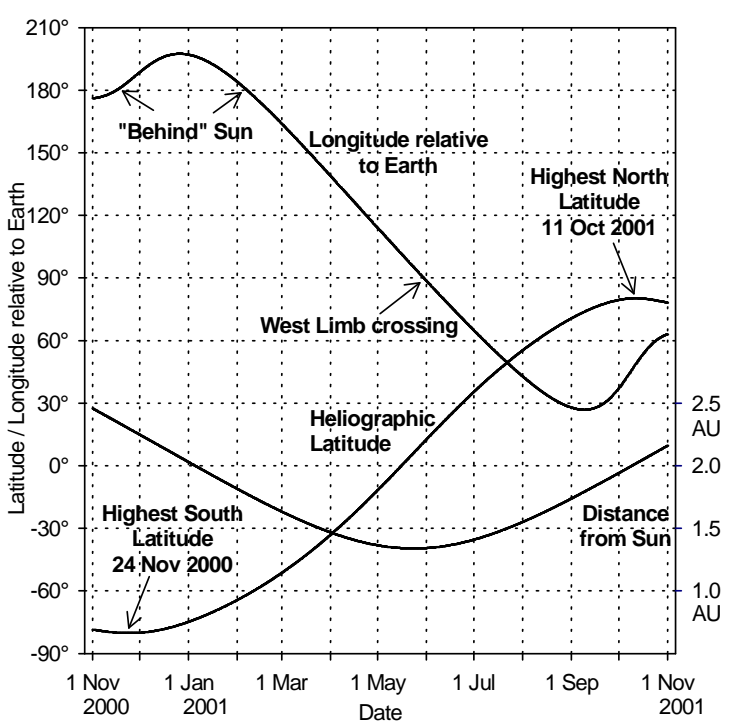

FIGURE 1. The trajectory of Ulysses through the fast latitude scan showing heliocentric distance, heliographic latitude and the longitude of the spacecraft relative to Earth. having a flux rope signature (FR), those marked with ' $\mathrm{x}$ ' (rather than '?') also exhibit the enhanced field magnitude and low proton temperature necessary to be classed as a magnetic cloud [8]. The driving of a shock wave is included as a signature since CIR shocks have typically not yet formed at the distances covered by Ulysses during this period. In addition, this paper represents the first time that both oxygen and iron charge state information have been combined together with both the field and plasma data in identifying ICMEs with Ulysses data. Studies of the systematic differences in the charge state distributions of heavier elements including oxygen [9] and iron [10] between ICMEs and the ambient solar wind are relatively recent. Their inclusion here is particularly interesting in view of the latitude range covered. The criteria for the charge state signatures were an average iron charge state greater than 12.0 and an $\mathrm{O}^{7+} / \mathrm{O}^{6+}$ ratio greater than 0.7. The charge state distributions are accumulated in 3 hour bins. We only included events where at least two contiguous bins satisfied the identification criteria. It should be noted that not all the signatures were present for the full duration of each event. The Start Time and End Time columns list the earliest possible start time and latest possible stop time obtained from combining all the signatures. These times are accurate to the nearest half hour at best due to the ICME signatures often not having sharp boundaries or alternatively due to the presence of multiple discontinuities, any of which could be the actual ICME boundary. The latitude quoted is that corresponding to the start time.

\section{Latitude Distribution of Events}

Figure 2 shows the locations in heliocentric distance and heliographic latitude of the ICMEs identified from Ulysses data during the solar maximum fast latitude scan (filled circles) and during the previous solar minimum fast latitude scan (open circle). It can be seen that during the solar minimum epoch only one ICME event was encountered during the $\sim 10$ month period [11], at a relatively low latitude of $23^{\circ} \mathrm{S}$ consistent with the latitude range covered by the streamer belt at that time. In contrast, we have identified 19 events during the equivalent solar maximum period, consistent with the $\mathrm{CME}$ rate being a strong function of solar activity.

These events are distributed over the full latitude range, in particular with ICMEs identified both close to $80^{\circ} \mathrm{S}$ and $80^{\circ} \mathrm{N}$. Although the figure does give an impression that there are fewer events at high latitudes, there are too few events to draw a firm conclusion 
TABLE 1. ICMEs Identified During the Ulysses Fast Latitude Scan.

\begin{tabular}{|c|c|c|c|c|c|c|c|c|c|c|}
\hline Start Time & End Time & Lat. & CSE & FR & Beta & Sh & $\mathbf{T p}$ & $\mathrm{He}$ & $\mathbf{F e}$ & O7/06 \\
\hline 20003410300 & 3450800 & $-79.6^{\circ}$ & $\bar{x}$ & $\mathrm{x}$ & $\bar{x}$ & & $\mathrm{x}$ & $\mathrm{x}$ & $?$ & \\
\hline 20010232300 & 0242030 & $-67.4^{\circ}$ & $\mathrm{x}$ & $\mathrm{x}$ & $\mathrm{x}$ & & $\mathrm{x}$ & & & \\
\hline 0321500 & 0341900 & $-64.0^{\circ}$ & $\mathrm{x}$ & $?$ & $\mathrm{x}$ & & $\mathrm{x}$ & & & \\
\hline 0770800 & 0782330 & $-41.7^{\circ}$ & $\mathrm{x}$ & $\mathrm{x}$ & $\mathrm{x}$ & $\mathrm{x}$ & $\mathrm{x}$ & & & \\
\hline 0800100 & 0810600 & $-40.0^{\circ}$ & & $\mathrm{x}$ & & & & $\mathrm{x}$ & $\mathrm{x}$ & $\mathrm{x}$ \\
\hline 0901800 & 0930800 & $-33.3^{\circ}$ & & & $\mathrm{x}$ & & $\mathrm{x}$ & $\mathrm{x}$ & & \\
\hline 1002100 & 1032100 & $-26.4^{\circ}$ & $\mathrm{x}$ & $\mathrm{x}$ & $\mathrm{x}$ & $\mathrm{x}$ & $\mathrm{x}$ & $\mathrm{x}$ & $\mathrm{x}$ & $\mathrm{x}$ \\
\hline 1041700 & 1060800 & $-23.5^{\circ}$ & $\mathrm{x}$ & & & $\mathrm{x}$ & & $?$ & $\mathrm{x}$ & $\mathrm{x}$ \\
\hline 1101000 & 1140800 & $-19.6^{\circ}$ & $\mathrm{x}$ & $\mathrm{x}$ & $\mathrm{x}$ & $\mathrm{x}$ & $\mathrm{x}$ & $\mathrm{x}$ & $\mathrm{x}$ & $\mathrm{x}$ \\
\hline 1300430 & 1330600 & $-4.5^{\circ}$ & $\mathrm{x}$ & $?$ & $\mathrm{x}$ & $\mathrm{x}$ & $\mathrm{x}$ & $\mathrm{x}$ & $\mathrm{x}$ & $\mathrm{x}$ \\
\hline 1390000 & 1420000 & $2.4^{\circ}$ & & & $\mathrm{x}$ & $\mathrm{x}$ & & $\mathrm{x}$ & & \\
\hline 1561100 & 1600400 & $16.2^{\circ}$ & $\mathrm{x}$ & $\mathrm{x}$ & & & $\mathrm{x}$ & $\mathrm{x}$ & $\mathrm{x}$ & $\mathrm{x}$ \\
\hline 1610500 & 1642330 & $20.0^{\circ}$ & $\mathrm{x}$ & $\mathrm{x}$ & $\mathrm{x}$ & & $\mathrm{x}$ & $\mathrm{x}$ & $\mathrm{x}$ & $\mathrm{x}$ \\
\hline 1771330 & 1791200 & $32.2^{\circ}$ & $\mathrm{x}$ & & & & & $\mathrm{x}$ & & \\
\hline 1851000 & 1881330 & $37.8^{\circ}$ & $\mathrm{x}$ & $\mathrm{x}$ & & & $\mathrm{x}$ & $\mathrm{x}$ & $\mathrm{x}$ & $\mathrm{x}$ \\
\hline 2041000 & 2082200 & $50.3^{\circ}$ & $\mathrm{x}$ & $\mathrm{x}$ & $\mathrm{x}$ & $\mathrm{x}$ & $\mathrm{x}$ & & & \\
\hline 2241800 & 2251600 & $61.8^{\circ}$ & $\mathrm{x}$ & & $\mathrm{x}$ & & & $\mathrm{x}$ & & \\
\hline 2361300 & 2370100 & $67.5^{\circ}$ & $\mathrm{x}$ & $\mathrm{x}$ & $\mathrm{x}$ & & $\mathrm{x}$ & $\mathrm{x}$ & & \\
\hline 2711230 & 2721630 & $79.1^{\circ}$ & $\mathrm{x}$ & $\mathrm{x}$ & $\mathrm{x}$ & & $\mathrm{x}$ & $\mathrm{x}$ & & \\
\hline
\end{tabular}

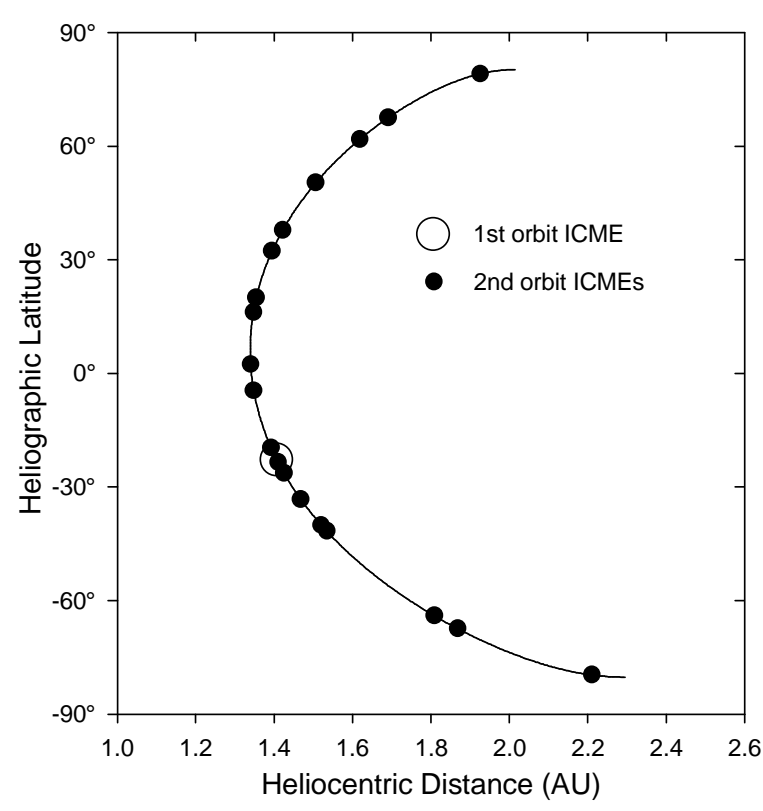

FIGURE 2. The locations of ICME events identified in Ulysses data during the fast latitude scans on both the first solar minimum orbit and the second solar maximum orbit.

from this data alone. However, when combined with events identified in 1999 and 2000 as Ulysses traversed high southerly latitudes prior to the fast latitude scan [12], it becomes clear that even at solar maximum, Ulysses has identified fewer events at high latitudes than at low to mid latitudes. This result is consistent with past results on the latitude distribution of CMEs obtained from coronagraph observations [2, 13] and with the present solar maximum observations by LASCO [O. C. St. Cyr, private communication].

\section{Solar Wind Context of Events}

During the solar maximum fast latitude scan the solar wind conditions were very different in the southern and northern polar regions [14]. In the south the solar wind was found to be a complex mixture of slow and intermediate speed streams whereas by the time Ulysses had reached the north polar regions fast solar wind from a newly reformed polar coronal hole had become re-established. The characteristics of the two highest latitude ICMEs observed in these different contexts were remarkably different. To illustrate this, a selection of the field and plasma parameters for the two events, the first and last from Table 1, are plotted in Figures 3 and 4. From top to bottom the six panels show the magnetic field magnitude $(|\mathbf{B}|)$, the two angles describing the magnetic field direction $\left(\phi_{B}, \theta_{B}\right)$, the solar wind speed $\left(v_{p}\right)$, the proton temperature $\left(T_{p}\right)$ and the alpha particle to proton ratio $(\alpha / p)$.

The highest southerly event (Figure 3 ) is similar in character to many other events observed by Ulysses at all latitudes at times when the solar wind is dominated by slow or intermediate speeds [e.g. 12]. The leading edge of the ICME can be seen to be propagating at a similar speed $(\sim 450 \mathrm{~km} / \mathrm{s})$ to the solar wind in front, thus the event is not driving a shock wave. The declining speed profile shows that the event is expanding as a result of the trailing solar wind being slower $(\sim 340 \mathrm{~km} / \mathrm{s})$. The magnetic cloud signature can be identified from the slow rotation seen in the two magnetic field angles coincident with a depression in the proton temperature. However, noting that the event 


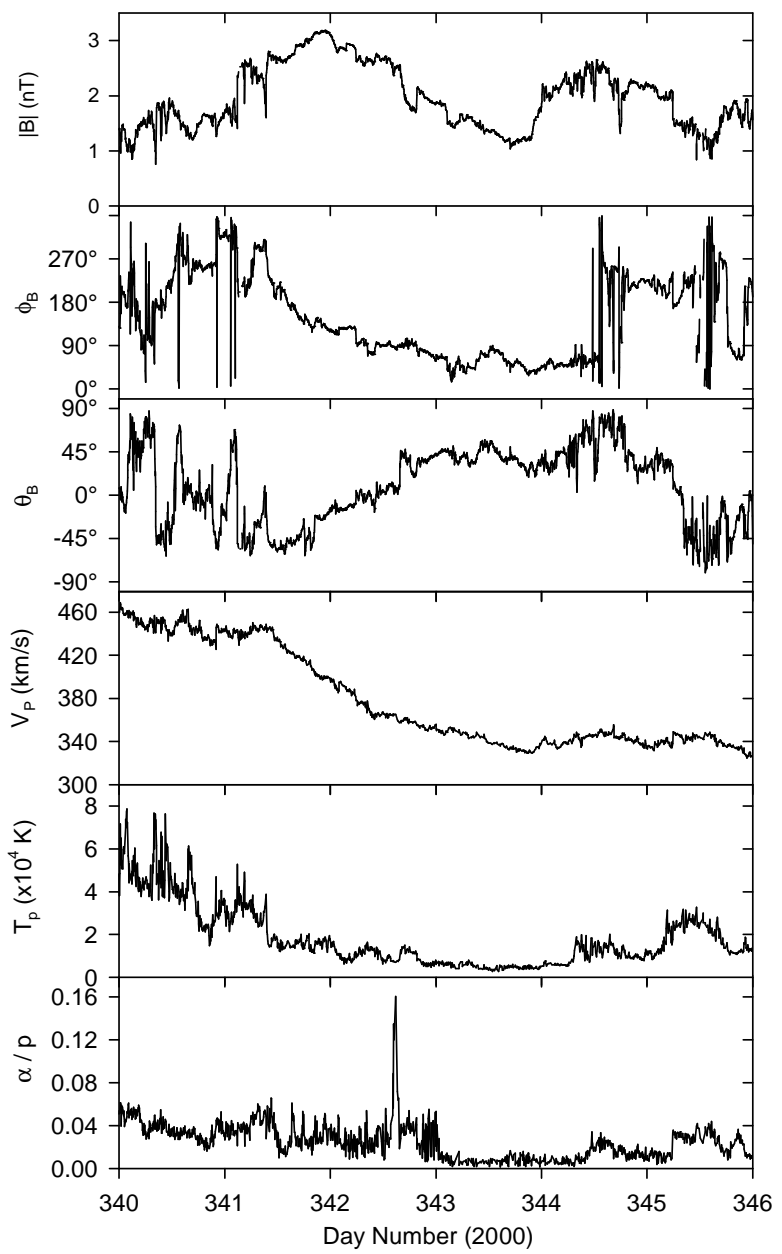

FIGURE 3. An example of an ICME observed by Ulysses at $79.6^{\circ} \mathrm{S}$. The panels are explained in the text.

continues into day 345, it can be seen that this ICME has a second part distinct from the magnetic cloud. This two (or more) part structure is typical of many of the ICMEs observed by Ulysses during the solar maximum period. It may be that the second part is a result of part of the same solar eruption with different properties or origin or due to a separate ICME immediately behind the first.

In contrast, the highest northerly event (Figure 4) has quite different characteristics. Here the solar wind speed both before and after the ICME event is fast $(\sim 750 \mathrm{~km} / \mathrm{s})$, showing the typical characteristics of solar wind originating from a polar coronal hole. The speed profile again indicates expansion, but here the expansion is due to the internal pressure of the ICME itself, typical of the over-expanded ICMEs previously observed embedded in the fast solar wind during the Ulysses first orbit closer to solar minimum [15]. Thus this type of ICME dynamic signature appears to be

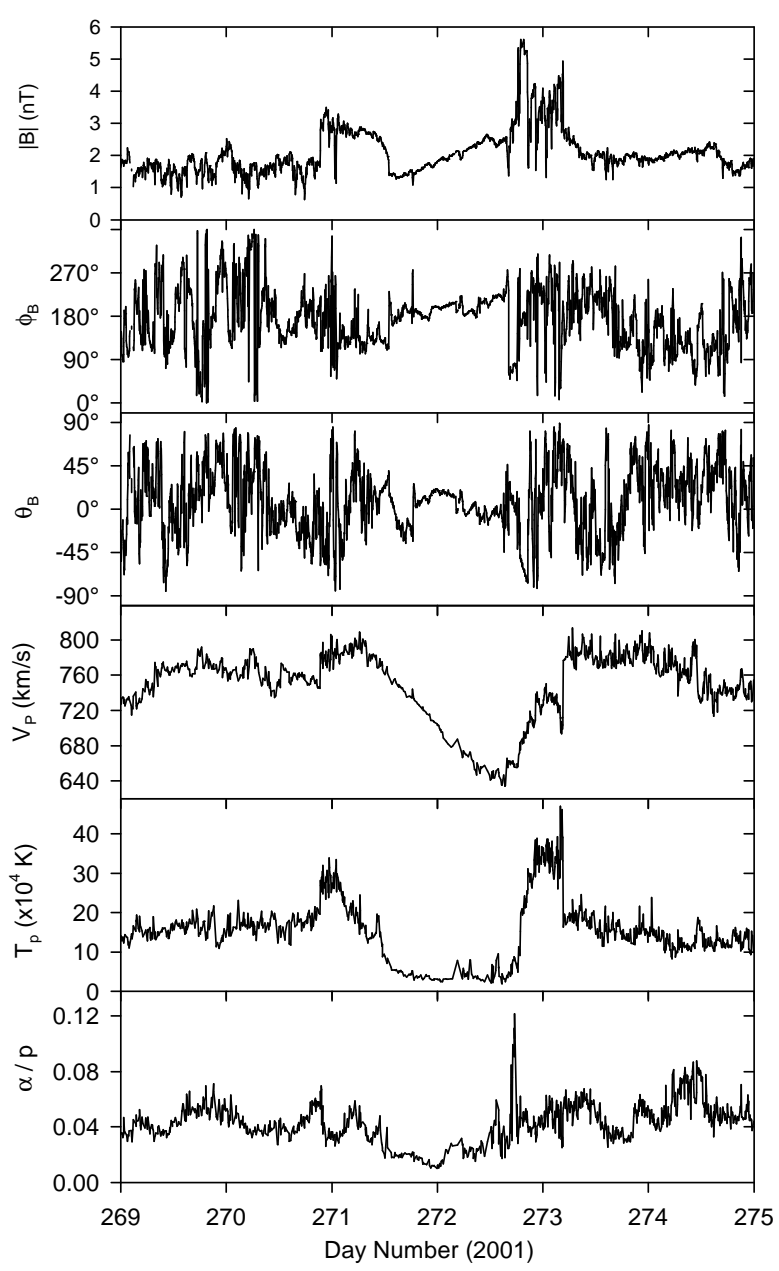

FIGURE 4. An example of an ICME observed by Ulysses at $79.1^{\circ} \mathrm{N}$. The panels are explained in the text.

unique to ICMEs propagating within high-speed solar wind streams [16]. This ICME also shows a magnetic cloud signature, indicating that magnetic clouds can be found at all latitudes at solar maximum. Previously at solar minimum, no magnetic clouds were observed at latitudes above $\sim 40^{\circ}$, even though ICME events were observed up to $\sim 60^{\circ}$.

\section{ICME Signatures}

In Table 1 we have provided an indication of the signatures exhibited by each of the fast latitude scan ICME events. In this section we give a first report on two specific results that are emerging from their study.

Firstly we comment on the latitude distribution and characteristics of those events showing the strongest iron and oxygen charge state signatures. It is notable that these events are confined within a latitude range 
within $40^{\circ}$ of the equator and are absent from the higher latitude events. All the well defined magnetic clouds in this latitude range show a clear difference in charge state distribution from the surrounding solar wind although the converse does not hold. This is similar to the results found for oxygen at solar minimum [9]. At that time there was an almost one-toone correspondence between magnetic clouds and anomalous composition signatures. These results suggest that this correlation does not hold for magnetic clouds observed at greater than $40^{\circ}$ from the equator. The reason for this is not obvious. It may be indicative of a recently suggested close association of these signatures with solar active regions and flares [17].

A second result that is emerging from this data concerns the axis orientation of the magnetic cloud events. This can be determined to a good approximation by fitting a force-free flux rope model to the magnetic field data [18]. The results show that axes of southern hemisphere magnetic clouds are predominantly directed in a westwards direction while those in the northern hemisphere are predominantly directed eastwards [A. Rees and R. J. Forsyth, manuscript in preparation]. Given that the data is obtained in an odd numbered solar cycle, this behavior is consistent with the predictions of [19] derived from near-ecliptic observations, dependent on the preferred orientations of prominences in the low solar atmosphere.

\section{CORRELATION WITH SOLAR OBSERVATIONS}

Returning to Figure 1, we now focus on the solar longitude of Ulysses with respect to that of the Earth. This is an important parameter for identifying periods when there should be good opportunities to compare CME images obtained by SOHO with in-situ observations from Ulysses. In particular, when this angle is $90^{\circ}$ Ulysses is in a prime position to intercept CMEs which erupt from the west limb of the Sun as seen from Earth, and Ulysses was within $30^{\circ}$ of this condition for a significant fraction of the fast latitude scan. Even during the period marked "Behind Sun" on Figure 1 when the longitude relative to Earth is $180^{\circ}$, at $80^{\circ} \mathrm{S}$ and $2 \mathrm{AU}$ from the Sun the spacecraft is still in a good position to intercept a subset of CMEs that appear in projection to erupt from the southern polar regions of the Sun. Using coronagraph observations to identify the particular solar event associated with an ICME event has been relatively successful close to solar minimum [e.g. 20], when the number of CMEs is relatively low. First attempts at a similar study with the recent Ulysses data have proved much harder due to confusion caused by the larger number of events. At high northern latitudes, immediately following the fast scan the solar sources of 3 out 5 ICMEs embedded in the fast solar wind were identifiable [16].

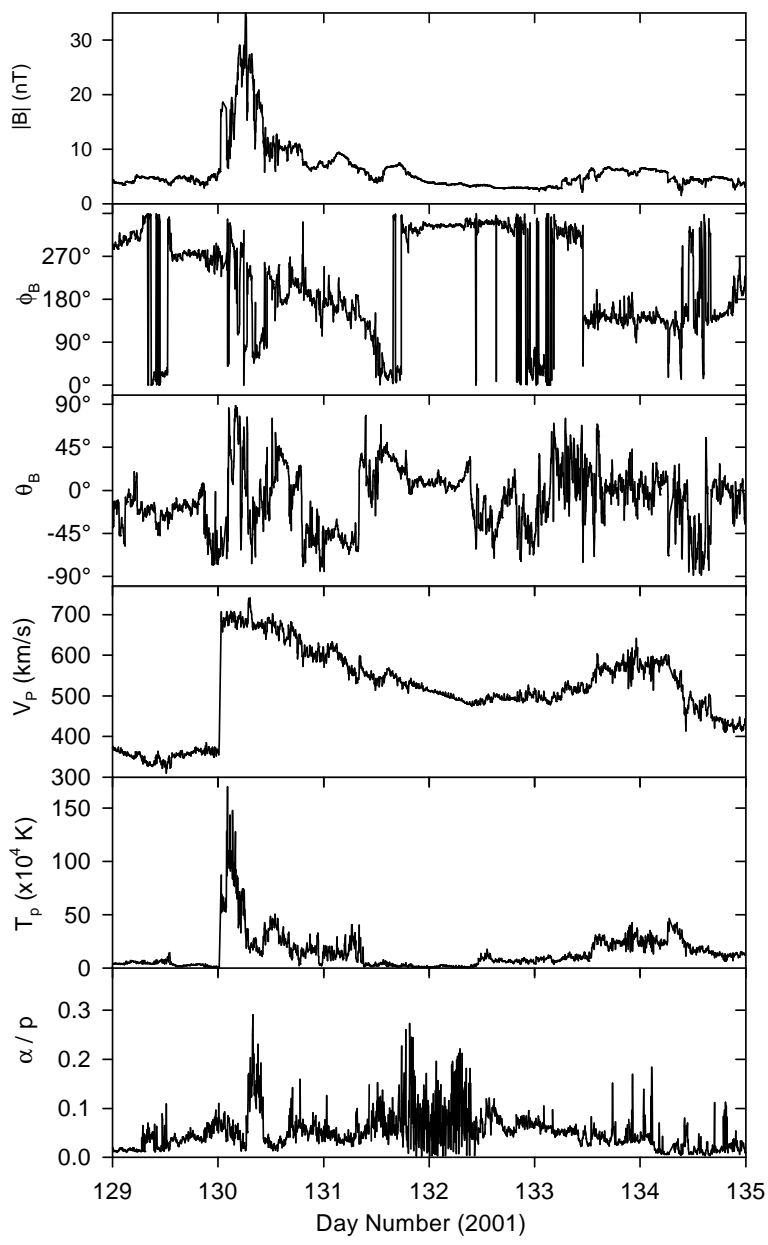

FIGURE 5. An example of an ICME observed by Ulysses near the equator associated with a west limb CME. The panels are the same as in Figures 3 and 4.

We conclude this paper by reporting on one dramatic west limb CME which occurred during the Ulysses fast scan where the solar to in-situ correlation was unmistakable. Figure 5 shows the in-situ signatures of this event, encountered by Ulysses on May 10 (day 130) 2001 at a latitude of $4.5^{\circ} \mathrm{S}$. The associated CME could be identified unambiguously as an eruption seen by LASCO on 2001 May 7 at 1206 UT which passed through the LASCO field of view at a speed of $\sim 1200 \mathrm{~km} / \mathrm{s}$ [http://cdaw.gsfc.nasa.gov/CME_list]. The time of arrival and speed of the ICME at Ulysses indicate that the ejecta was being decelerated as it 
interacted with slower solar wind as it traveled out to Ulysses. The interaction produced an unusually strong shock wave leading the event as it passed Ulysses. The magnetic field strength of $\sim 35 \mathrm{nT}$ was the highest recorded by the Ulysses magnetometer in interplanetary space during the 11 year mission so far. The LASCO images show a complex multi-part eruption while the leading part of the event at Ulysses consisted of many field rotations and current sheets. It is likely that the complexity seen at Ulysses is due both to the structures of the original $\mathrm{CME}$ and the strong compression that has clearly taken place during the journey out from the Sun. Analysis of the planar structuring of the discontinuities in the sheath region ahead of the ICME suggests that the bulk of the ejecta was located at more positive longitudes relative to Ulysses [21], that is further behind the limb as seen from the Earth. It was not possible to identify a clear magnetic cloud in the leading part of the event but the low beta and high helium content clearly indicate the presence of magnetic cloud-like material.

\section{SUMMARY}

We have presented an overview of the ICME events observed by Ulysses during the solar maximum fast latitude scan, cataloguing their occurrence and signatures, as well as presenting three example events. Clearly there is much scope for further work with this data set, for example on the changes noted in the composition signatures with latitude, and for a more detailed correlative study of the Ulysses events with those observed by LASCO in the same time period.

\section{ACKNOWLEDGMENTS}

Ulysses research at Imperial College London is supported by the UK Particle Physics and Astronomy Research Council. Work at Los Alamos was performed under the auspices of the US Department of Energy with support from NASA.

\section{REFERENCES}

1. Webb, D. F., and Howard, R. A., J. Geophys. Res. 99, 4201-4220 (1994).

2. Hundhausen, A. J., J. Geophys. Res. 98, 13177-13200 (1993).
3. Brueckner, G. E., Howard, R. A., Koomen, M. J., et al., Solar Phys. 162, 357-402 (1995).

4. Balogh, A., Beek, T. J., Forsyth, R. J., et al., Astron. Astrophys. Suppl. Ser. 92, 221-236 (1992).

5. Bame, S. J., McComas, D. J., Barraclough, B. L., et al., Astron. Astrophys. Suppl. Ser. 92, 237-266 (1992).

6. Gloeckler, G., Geiss, J., Balsiger, $\mathrm{H}$ et al., Astron. Astrophys. Suppl. Ser. 92, 267-290 (1992).

7. Neugebauer, M., and Goldstein, R., "Particle and field signatures of coronal mass ejections in the heliosphere," in Coronal Mass Ejections, edited by N. Crooker, J. A. Joselyn, and J. Feynman, Washington D. C.: AGU, 1997, pp. 245-251.

8. Burlaga, L. F., "Magnetic clouds," in Physics of the inner heliosphere II, edited by R. Schwenn and E. Marsch, Berlin: Springer-Verlag, 1991, pp. 1-22.

9. Henke, T., Woch, J., Mall, U., et al., Geophys. Res. Lett. 25, 3465-3468 (1998).

10. Lepri, S. T., Zurbuchen, T. H., Fisk, L. A., et al., J. Geophys. Res. 106, 29231-29238 (2001).

11. Gosling, J. T., Bame, S. J., Feldman, W. C., et al., Geophys. Res. Lett. 22, 3329-3332 (1995).

12. Gosling, J. T., and Forsyth, R. J., Space Sci. Rev. 97, 8798 (2001).

13. St. Cyr, O. C., Howard, R. A., Sheeley, N. R., et al., J. Geophys. Res. 105, 18169-18185 (2000).

14. McComas, D. J., Elliott, H. A., Gosling, J. T., et al., Geophys. Res. Lett. 29, 10.1029/2001GLO14164 (2002).

15. Gosling, J. T., Riley, P., McComas, D. J., and Pizzo, V. J., J. Geophys. Res. 103, 1941-1954 (1998).

16. Reisenfeld, D. B., Gosling, J. T., Steinberg, J. T., et al., in Proceedings of Solar Wind 10, this volume.

17. Richardson, I. G., Cane, H. V., Lepri, S. T., et al., in Proceedings of Solar Wind 10, this volume.

18. Lepping, R. P., Jones, J. A., and Burlaga, L. F., J. Geophys. Res. 95, 11957-11965 (1990).

19. Bothmer, V., and Schwenn, R., Ann. Geophys. 16, 1-24 (1998).

20. Funsten, H. O., Gosling, J. T., Riley, P., et al. J. Geophys. Res. 104, 6679-6689 (1999).

21. Jones, G. H., Rees, A., Balogh, A., and Forsyth, R. J., Geophys. Res. Lett. 29, 10.1029/2001GL014110 (2002). 